\title{
Immunophenotyping Pattern in Childhood Acute Leukemia in the Adam Malik Hospital Medan
}

\author{
Putri Chadijah Tampubolon${ }^{1}$, Bidasari Lubis ${ }^{2}$, Adi Koesoema Aman ${ }^{1}$, Malayana R Nasution $^{1}$ \\ ${ }^{1}$ Department of Clinical Pathology, Faculty of Medicine, Sumatera Utara University/Adam Malik Hospital, Medan, Indonesia. E-mail: \\ chadijah.putridr@gmail.com \\ ${ }^{2}$ Department of Pediatrics, Faculty of Medicine, Sumatera Utara University/Adam Malik Hospital, Medan, Indonesia
}

\begin{abstract}
Leukemia is the most common malignancy at the age of under 15 years, with a ratio of 1 to 3 cancer cases in children. Immunophenotyping will improve accuracy and easily provide data reproducibility. To determine the immunophenotyping pattern in patients with acute leukemia in the Pediatric Center at the Adam Malik General Hospital, Medan. This research was a cross-sectional study in children suffering from acute leukemia in the Pediatric Unit Adam Malik General Hospital, Medan based on $\mathrm{CBC}$, peripheral smear, bone marrow morphology, and flow cytometry immunophenotyping. Samples were evaluated for blast morphologic and immunophenotyping was carried out. Results of morphologic observation and immunophenotyping were compared. From 20 samples using the monoclonal antibody with flow cytometry immunophenotyping, concordance with morphology is good $(\mathrm{K}=0.886)$. After classification, the percentage of acute leukemia was $45 \%$ B-ALL, 35\% AML, and 20\% T-ALL. One of 10 samples morphologically classified as ALL but reported as AML. Immunophenotyping has been shown to increase diagnostic accuracy and assist in establishing lines in blast cells, which was initially merely based on morphological features.
\end{abstract}

Keywords: Aberrant, acute leukemia, flow cytometry, immunophenotyping

\section{INTRODUCTION}

Leukemia is the most common malignancy at the age of under 15 years, with a ratio of 1 to 3 cancer cases in children. The two main subtypes of leukemia found in children are Acute Lymphoblastic Leukemia (ALL) and Acute Myeloid Leukemia (AML), with a percentage of $80 \%$ and $17 \%$, respectively. Childhood leukemia continues to be a global public health problem because the incidence of this malignancy seems to continue to increase. The peak incidence occurs at age of $2-5$ years and the average incidence of children under the age of 15 is $4-4.5 / 100,000$ peryear. ${ }^{1,2}$

The diagnosis of acute leukemia requires the integration of hematopathological diagnosis depending on studies of cell morphology, application of immunophenotyping and cytogenetic flow cytometry according to WHO 2016

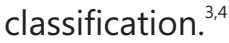

Immunophenotyping analysis with multiparameter flow cytometry will determine the characteristics of malignant blood cells with antigen differentiation that appears at various stages of hematopoietic development and the lineage involved by the expression of specific CD markers.
Differences in expression of surface membrane antigens and cytoplasmic components are used to identify and classify cell origin and stage of differentiation from acute leukemia. ${ }^{3-6}$

Immunophenotyping will increase accuracy and easily provide data reproducibility of the Acute Leukemia. Immunophenotyping can be used to identify the lineage of acute leukemia and classify it into T-ALL, B-ALL, or AML (myeloid lineage). This is important because the management and prognosis of the diseases are different. ${ }^{3,5-7}$

Aberrant antigen expression can also be detected through flow cytometry immunophenotyping and is useful for diagnostic, prognostic, and clinical management. This is in accordance with studies by Ossenokopele et al. ${ }^{7.8}$

Data in Indonesia regarding the usage of immunophenotyping in acute leukemia as diagnostic instruments remain limited. From this phenomenon, this study aimed to determine the immunophenotyping pattern of pediatric patients with acute leukemia. It was expected that this study can be used as a reference for clinicians in diagnostic accuracy and prognostic acute leukemia. It was expected that in the future the implementation of immunophenotyping can become a diagnostic 
standard and help establish the diagnosis of acute leukemia following with WHO standards. ${ }^{6,79}$

\section{METHODS}

Bone marrow aspirates from 20 patients aged 0-17 years suspected of acute leukemia were used as samples in this study. This study was performed between April and October 2019 at the Adam Malik General Hospital in Medan. The sample was newly collected sample and was analyzed at the latest within 24 hours. The three-color flow cytometric method in this study used a monoclonal antibody (MoAb) with 14 sets of leukemia panels (Table 1$)$. The morphological observation was carried out on peripheral blood and bone marrow aspirates.

Table 1. Monoclonal antibody panel

\begin{tabular}{ll}
\hline Lineage & MoAb \\
\hline B lineage & CD10, CD19, CD20 \\
T lineage & CD3, CD5, CD7 \\
Myeloid lineage & CD13, CD14, CD33, \\
& CD117, HLA DR \\
Non-lineage & CD34, CD45 \\
\hline
\end{tabular}

Sample preparation for immunophenotyping was started with the lyse/wash protocol and $50 \mu \mathrm{L}$ of the sample was then put into a Falcon tube and $10 \mu \mathrm{L}$ of monoclonal antibody was added. The sample was then incubated in a dark room for 30 minutes and the lysing solution was added and re-incubated for 15 minutes. The sample was then centrifuged at $500 \mathrm{~g}$ for 10 minutes. The supernatant was removed and the precipitate was washed with phosphate buffer solution, re-centrifuged, and the supernatant was then removed. The sample was then read using the BD FACS Calibur.

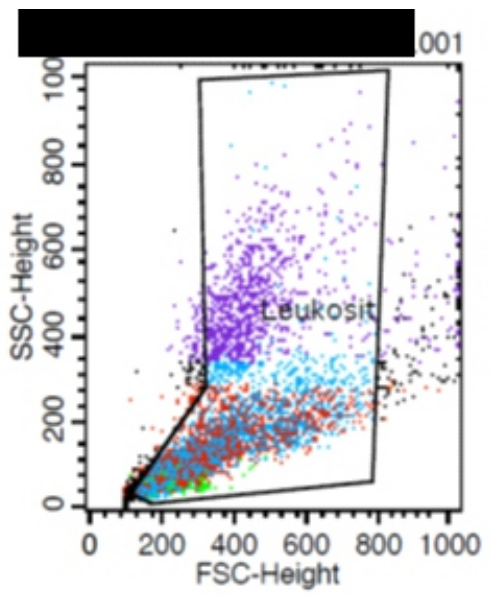

Fluorescents used in this study were Peridinin-chlorophyll protein complex (PerCP), Fluorescein Isothiocyanate (FITC), Phycoerythrin (PE), and Allophycocyanin (APC).

CD45 gating was used for the population of leukocytes along with side scatter for immunophenotyping analysis of acute leukemia. The CD34 marker was used for blast cells. Studies have shown that a gating strategy using CD34 expression is the most superior marker for identification of leukemia cell populations in either peripheral blood or marrow specimens in leukemia (Figure 1).

Phenotypes were classified based on reactions to monoclonal antibodies and the type of blast was then determined and classified into B-ALL, T-ALL, and AML (Table 1). In some cases, co-expression from different lineage markers are found and expressed as aberrant phenotypes. Aberrant phenotype or a deviant phenotype is a phenomenon in which lymphoid-related markers or myeloid-related markers are expressed together in myeloblasts and lymphoblasts. Distorted phenotypic events have been reported in ALL and AML with varying frequencies and prognostic values, which remain controversial. In a recent study, deviant myeloid expression with $\mathrm{CD} 13$ and/or CD33 antigens was reported in $35 \%$ of ALL cases. In some studies, the aberrant phenotype was found to have a significant prognostic, despite no deviating differences between aberrant phenotype and the normal phenotype were found in other studies.

Research permission was obtained from the Health Research Ethics Committee of the Faculty of Medicine, Sumatera Utara University/General Hospital Adam Malik Medan, with number 536/TGL/KEPK FK USU-RSUP HAM/2019.

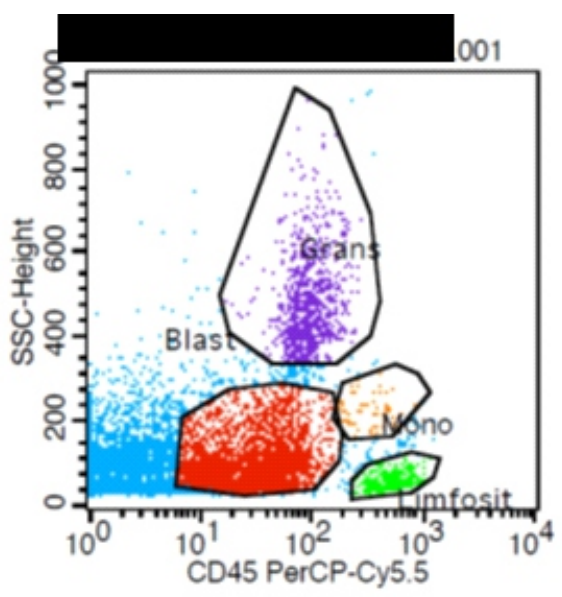

Figure 1: Left: Cell population-based on forward scatter and side scatter. Right: Leukocytes populations were marked with CD45 markers and grouped into blasts (red), monocytes (orange), lymphocytes (green), granulocytes (purple). Blast cell gating showed CD45 dim with low side scatter 


\section{RESULTS AND DISCUSSION}

Samples from 20 patients aged 0-17 years were classified based on immunophenotyping. The patient classification can be seen in Figure 2. Patient characteristics can be seen in Table 2 and the hematologic profile can be seen in Table 3 .

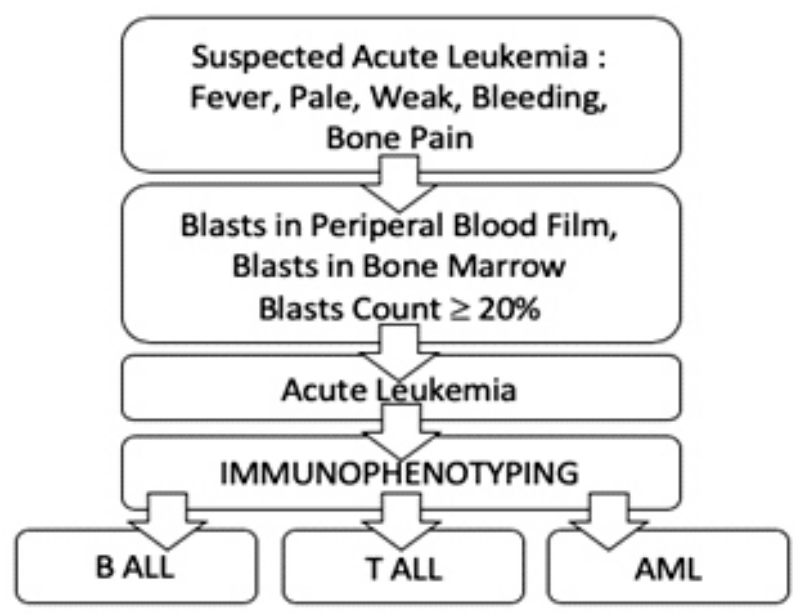

Figure 2. Flowchart of acute leukemia classification in this study

Table 3 showed the average leukocyte count of $39.16 \times 10^{3} / \mu \mathrm{L}$ and $55.86 \times 10^{3} / \mu \mathrm{L}$ in patients with ALL and $A M L$, respectively. These results were higher than the normal range of leukocyte counts. The mean platelet count in patients with ALL and AML was $60.78 \times 10^{3} / \mu \mathrm{L}$ and $44.97 \times 10^{3} / \mu \mathrm{L}$, respectively, showing lower results compared to the normal range of platelet counts with a $p$-value of 0.778 . The mean values of $\mathrm{Hb}$ levels in patients with $\mathrm{ALL}$ and $\mathrm{AML}$ were $7.86 \mathrm{~g} / \mathrm{dL}$ and $8.31 \mathrm{~g} / \mathrm{dL}$, respectively ( $p$-value of $0.699)$.

Table 2. Patient characteristics

\begin{tabular}{lc}
\hline \multicolumn{1}{c}{ Variables } & Patients (n=20) \\
\hline Age & \\
$<5$ years & $4(20 \%)$ \\
$5-9$ years & $8(40 \%)$ \\
$10-14$ years & $3(15 \%)$ \\
15-17 years & $5(25 \%)$ \\
Gender & \\
$\quad$ Male & $10(50 \%)$ \\
$\quad$ Female & $10(50 \%)$ \\
Lymphadenopathy & \\
$\quad$ Palpable & $6(40 \%)$ \\
$\quad$ None & $14(60 \%)$ \\
Splenomegaly/Hepatomegaly & \\
$\quad$ Palpable & $11(55 \%)$ \\
$\quad$ None & $9(45 \%)$ \\
Anemia (Hb < 10g/dL) & \\
$\quad$ Found & $16(80 \%)$ \\
$\quad$ None & $4(20 \%)$ \\
Low platelet (platelet < 100.000) & \\
$\quad$ Found & $15(75 \%)$ \\
$\quad$ None & $5(25 \%)$ \\
Leukocytosis (WBC > 50.000) & \\
$\quad$ Found & $7(35 \%)$ \\
$\quad$ None & $13(65 \%)$ \\
Morphology & \\
ALL & \\
AML & $6(30 \%)$ \\
\hline
\end{tabular}

Table 3. Hematologic profile

\begin{tabular}{|c|c|c|c|}
\hline Parameter Frequency (\%) & ALL $n=14$ & AML $n=6$ & p-value \\
\hline \multicolumn{4}{|l|}{ White blood count $\left(\times 10^{3} / \mu \mathrm{L}\right)$} \\
\hline Mean $\pm S D$ & $39.16 \pm 54.02$ & $55.86 \pm 55.05$ & $0.187^{*}$ \\
\hline Median & $9.14(16.00-185,02)$ & $31.61(8.18-143,51)$ & \\
\hline \multicolumn{4}{|l|}{$\mathrm{Hb}(\mathrm{g} / \mathrm{dL})$} \\
\hline Mean \pm SD & $7.86 \pm 2.45$ & $8.31 \pm 2.31$ & $0.699^{* *}$ \\
\hline Median & $8.45(3.20-10.60)$ & $8.45(4.50-11.60)$ & \\
\hline \multicolumn{4}{|l|}{ Platelet $\left(\times 10^{3} / \mu \mathrm{L}\right)$} \\
\hline Mean \pm SD & $60.78 \pm 47.23$ & $54.33 \pm 44.97$ & $0.778^{* *}$ \\
\hline Median & $60.50(7.00-150)$ & $56.00(11.00-115,00)$ & \\
\hline \multicolumn{4}{|l|}{ Blast count in peripheral blood (\%) } \\
\hline Mean \pm SD & $26.64 \pm 22.59$ & $32.83 \pm 21.21$ & $0.560^{*}$ \\
\hline Median \pm SD & $30.00(0-60)$ & $38.00(0-56.00)$ & \\
\hline \multicolumn{4}{|l|}{ Blast count in bone marrow (\%) } \\
\hline Mean \pm SD & $52.80 \pm 22.07$ & $61.91 \pm 21.62$ & $0.406^{* *}$ \\
\hline Median \pm SD & $49.62(17.00-95)$ & $66.50(0-90)$ & \\
\hline
\end{tabular}

*Mann-Whitney **T-test 
Table 4 showed that B-ALL was the most commonly found acute leukemia (45\%) followed by AML (35\%) and T-ALL (20\%).

Table 4. Results of flow cytometry immunophenotyping using BD FACS Calibur

\begin{tabular}{lc}
\hline Immunophenotyping & Sample \\
\hline B-ALL & $9(45 \%)$ \\
T-ALL & $4(20 \%)$ \\
Myeloid lineage & $7(35 \%)$ \\
Total & $20(100 \%)$ \\
\hline
\end{tabular}

Figure 6 showed that complete remission was the most common outcome in patients with B-ALL (55.55\%), followed by partial remission in patients with myeloid lineage (28.58\%), and death in patients with T-ALL (75\%).

From 14 patients diagnosed with ALL based on morphological observation, one was reported as AML by immunophenotyping. Contrastingly, patients formerly diagnosed with $A M L$ were all reported as $\mathrm{AML}$ using immunophenotyping, indicating a concordance between both methods $(\mathrm{K}=0.886)$.
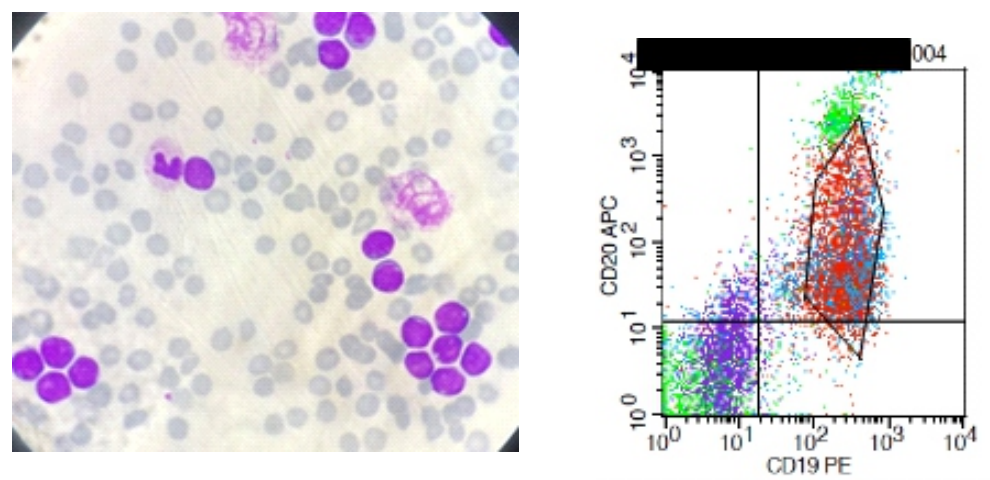

Figure 3. Left: Blast cells appear in peripheral blood smear preparations of patients with suspected acute leukemia. Right: CD19 and CD20 expression in the blasts of the patients with B lineage ALL
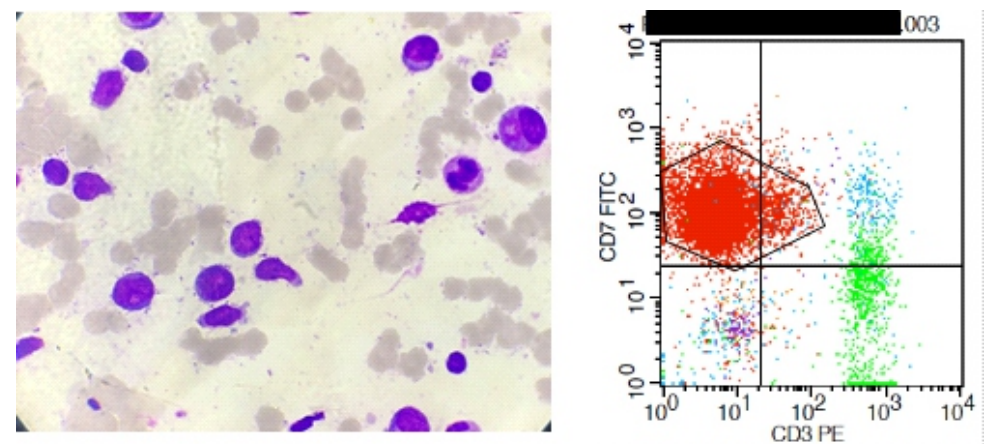

Figure 4. Left: Lymphoblasts appear on bone marrow smears. Right: CD7, CD3 expression in the blasts of the patient with T lineage ALL
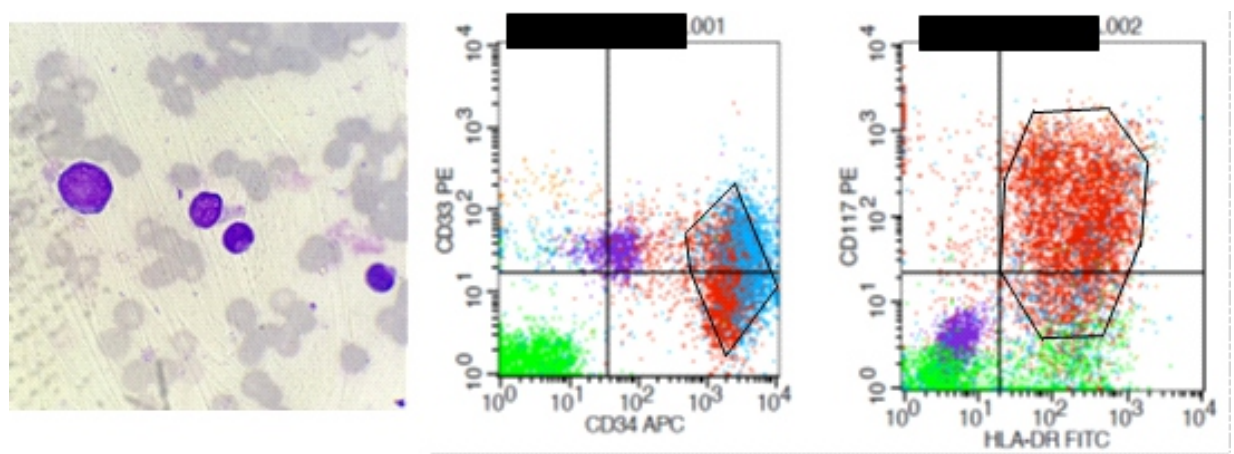

Figure 5. Left: Lymphoblasts appear on bone marrow smears. Right: CD33, CD117, HLA DR expression in blasts of patients with ALL showing myeloid lineage 


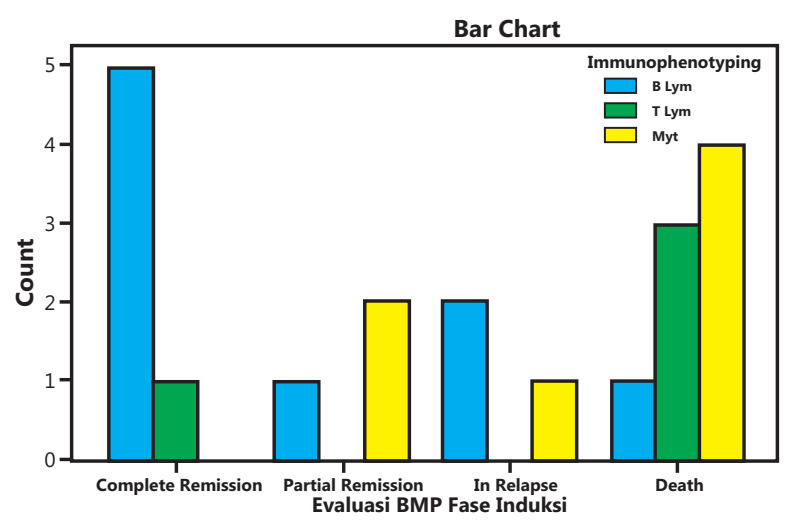

Figure 6. Immunophenotyping patterns and therapeutic response after chemotherapy induction

Table 5. Concordance between morphology and immunophenotyping

\begin{tabular}{llll}
\hline \multirow{2}{*}{ Morphology } & \multicolumn{3}{c}{ Immunophenotyping } \\
\cline { 2 - 4 } & ALL & AML & Total \\
\hline ALL & 13 & 1 & 14 \\
AML & 0 & 6 & 6 \\
Total & 13 & 7 & 20 \\
\hline
\end{tabular}

In this study, it was found that patients with acute leukemia with an age range of 5-9 years were most affected by acute leukemia with a percentage of $40 \%$, according to research by Supriyadi et al. ${ }^{3,10}$ It was found in this study that gender did not affect the risk of acute leukemia with the equal number of cases (50\%). This was different from research by Sharma, which showed said that boys had a higher risk of acute leukemia compared to girls. ${ }^{11}$ Thirty-five percent leukocytosis (leukocytes $>50 \times 10^{3} / \mu \mathrm{L}$ ) was found. This is important in determining risk stratification in patients with acute leukemia, especially in ALL, that the state of leukocytosis (leukocytes $>50 \times 10^{3} / \mu \mathrm{L}$ ), the age of patients $>10$ years and male gender has a worse prognosis compared to those without these criteria or what is known as high-risk. ${ }^{12,13}$

The most common type of acute leukemia in this study was ALL with a total of $70 \%$ cases. This result was in accordance with the previous study by Kamps et al. and Milne et al. Also, total cases of anemia and thrombocytopenia in this study were $80 \%$ and $75 \%$, respectively. Further observation was needed because it has been linked to the state of bone marrow failure, which is also a poor prognosis of acute leukemia. ${ }^{14}$

Leukocyte counts were higher in patients with AML compared to patients with ALL in this study, suggesting an association with a worse prognosis. Studies showed delayed treatment of leukocytosis may increase mortality by up to $40 \% .{ }^{15}$

There was no significant difference in $\mathrm{Hb}$ levels in patients with ALL and AML with p-value $=0.699$. Similarly, there was no significant difference in platelet count between ALL and AML patients with $p$-value $=0.778$. Also, there was no significant difference in blast count in the bone marrow aspirate of patients with ALL and patients with AML ( $p$-value $=0.406)$.

B-ALL was the most common type of acute leukemia (45\%) followed by AML (35\%) and T-ALL (20\%), similar to a study by Supriyadi, Chiaretti, Gajendra, and Van. ${ }^{2,3,6,7}$ B-ALL is acute leukemia with the best prognostic with a cure rate of $95 \%{ }^{8,10,16,17}$

Complete remission was found in patients with B-ALL (55.55\%), the highest number compared to other types of leukemia. Further study was needed to assess the prognostic of patients with leukemia using immunophenotyping. Besides, partial remission was the most common outcome in patients with myeloid lineage (28.58\%) and death was the most common outcome in patients with T-ALL (75\%). This was consistent with studies by Vaitkeviciene et al ${ }^{18,19}$

As for the aberrant phenotype, CD13 was frequently found in B-ALL (22.22\%), CD33 in T-ALL (50\%), and CD19 in myeloid lineage (42.8\%). These findings were also reported in studies by tha et al. ${ }^{9}$ If related to the response to chemotherapy induction, there was no difference between aberrant CD13 myeloid antigen on B-ALL and B-ALL without aberrant antigen with the same level of complete remission (33.33\%). Besides, 37.5\% death and 28.58\% partial remission were found in patients with AML with an aberrant CD19 and patients with AML without an aberrant antigen, respectively.

From 14 patients who were morphologically diagnosed with ALL, one was reported as AML by immunophenotyping. Whereas patients with $A M L$ were all reported as $A M L$ using immunophenotyping, with good morphological concordance $(\mathrm{K}=0.886)$. One sample was diagnosed with ALL based on morphological observation but reported as AML by immunophenotyping, with both myeloid and lymphoid were found in this sample. Further examination was required to establish a mixed phenotype acute leukemia state. However, a study by Belurkar et al. showed that a mixed lineage leukemia condition can be established if blast cells $>50 \%$, which express lymphoid markers (CD19 and CD20) and myeloid markers (CD33 and CD13) are found. ${ }^{20}$ 


\section{CONCLUSION AND SUGGESTIONS}

There was no significant difference in hemoglobin levels and platelet count in patients with $A L L$ and patients with AML $(p>0.05)$. B-ALL was the most common type of acute leukemia (45\%) followed by AML (35\%) and T-ALL (20\%). CD13 is an aberrant phenotype, which was the most common in B-ALL (22.22\%), CD33 in T-ALL (50\%), and CD19 in the myeloid lineage (42.8\%).

Complete remission was the most common outcome in patients with B-ALL (55.55\%), Partial remission was the most common outcome in patients with myeloid lineage $(28.58 \%)$, and death was the most common outcome in patients with T-ALL (75\%). Concordance between morphology and immunophenotyping was moderately good $(\mathrm{K}=0.886)$. Immunophenotyping is an effective instrument to increase diagnostic accuracy and assist in the process of establishing lines in blast cells according to WHO diagnostic standards.

Cytoplasmic markers need to be added in cases with suspected mixed phenotype acute leukemia. Further research analyzing the relationship of immunophenotyping patterns to determine the prognostic of acute leukemia was needed.

\section{REFERENCES}

1. Permono B, Ugrasena I. Leukimia akut. In: Permono B, Sutaryo, Ugrasena, Windiastuti E, Abdulsalam M. $3^{\text {rd }}$ Ed., Jakarta, Ikatan Dokter Anak Indonesia, 2010; 236-47.

2. Demanelis K. Differences in childhood leukemia incidence and survival between Southern Thailand and the United States: A population-based analysis. Pediatr Blood Cancer, 2016; 62(10): 1790-8.

3. Supriyadi E, Widjajanto PH, Veerman AJP, Purwanto I, Nency YM, et al. Immunophenotypic patterns of childhood acute leukemias in Indonesia. Asian Pacific J Cancer Prev, 2011; 12(12): 3381-7.

4. Swerdlow SH, Campo E, Pileri SA, Harris NL, Stein $\mathrm{H}_{\text {, }}$ et al. Introduction to WHO classification of tumours of haematopoietic and lymphoid tissues in WHO classification of tumours of haematopoietic and lymphoid tissues. Perancis, International Agency for Research on Cancer (IARC), 2016; 13-14.

5. Chiaretti S, Zini G, Bassan R. Diagnosis and subclassification of acute lymphoblastic leukemia. Mediterr J Hematol Infect Dis, 2014; 6(1): e2014073.

6. Van Dongen JJM, Lhermitte L, Böttcher S, Almeida J, Van Der Velden VHJ, et al. EuroFlow antibody panels for standardized n-dimensional flow cytometric immunophenotyping of normal, reactive and malignant leukocytes. Leukemia, 2012; 26(9): 1908-75.

7. Gajendra S. Flow cytometry in acute leukemia. Clin Oncol, 2016; 1: 1-5.
8. Ossenkoppele GJ, van de Loosdrecht AA, Schuurhuis GJ. Review of the relevance of aberrant antigen expression by flow cytometry in myeloid neoplasms. Br J Haematol, 2011; 153(4): 421-36.

9. Jha R, Grover G, Bose P. Lymphoid associated antigen expression in new cases of Acute Myeloid Leukemia. J Pathol Nepal, 2013; 3(6): 487-90.

10. Boissel N, Auclerc MF, Lhéritier V, Perel Y, Thomas $X$, et al. Should adolescents with acute lymphoblastic leukemia be treated as old children or young adults? Comparison of the French FRALLE-93 and LALA-94 trials. J Clin Oncol. 2003; 21(5): 774-80.

11. Sharma M, Sachdeva M, Varma N, Varma $S$, Marwaha R. Characterization of immunophenotypic aberrancies in adult and childhood acute lymphoblastic leukemia: A study from Northern India. J Cancer Res Ther, 2016; 12(2): 620-6.

12. Bhojwani D, Howard SC, Pui CH. High-risk childhood acute lymphoblastic leukemia. Clin Lymphoma Myeloma [Internet]. 2009; 9(suppl 3): S222-30. Available from: http://dx.doi.org/10.3816/CLM. 2009.s.016 (accessed 10 Nov, 2019).

13. Schiffer CA, Connor RF. Hyperleucocitosis and leukostasis in hematologic malignances. UpToDate [Internet]. 2016; 1-9. Available from: www.uptodate.com (accessed 10 Nov, 2019).

14. Terwilliger $T$, Abdul-Hay M. Acute lymphoblastic leukemia: A comprehensive review and 2017 update. Blood Cancer J, 2017; 7(6): e577.

15. Giammarco S, Chiusolo P, Piccirillo N, Di Giovanni A, Metafuni $E$, et al. Hyperleukocytosis and leukostasis: management of a medical emergency. Expert Rev Hematol [Internet]. 2017; 10(2): 147-54. Available from: http://dx.doi.org/10.1080/17474086.2017. 1270754 (accessed 10 Nov, 2019).

16. Borowitz MJ. B-lymphoblastic leukaemia/lymphoma, not otherwise specified (NOS). Introduction to WHO classification of tumours of haematopoietic and lymphoid tissues in WHO classification of tumours of haematopoietic and lymphoid tissues. 2017; 204.

17. Hallböök H, Gustafsson G, Smedmyr B, Söderhäll S, Heyman M. Treatment outcome in young adults and children > 10 year of age with acute lymphoblastic leukemia in Sweden: A comparison between a pediatric protocol and an adult protocol. Cancer, 2006; 107(7): 1551-61.

18. Vaitkeviciene $G$, Heyman M, Jonsson OG, Lausen $B$, Harila-Saari A, et al. Early morbidity and mortality in childhood acute lymphoblastic leukemia with very high white blood cell count. Leukemia, 2013; 27(11): 2259-62.

19. Teachey DT, Hunger SP. Predicting relapse risk in childhood acute lymphoblastic leukaemia. $\mathrm{Br} \mathrm{J}$ Haematol, 2013; 162(5): 606-20.

20. Belurkar S, Mantravadi H, Manohar C, Kurien A. Correlation of morphologic and cytochemical diagnosis with flowcytometric analysis in acute leukemia. J Cancer Res Ther, 2013; 9(1): 71-9. 